\title{
Effective Control of Chemical Potentials by Rabi Coupling with RF-Fields in Ultracold Mixtures
}

\author{
Luca Lepori ${ }^{1,2, *}$, Anna Maraga ${ }^{3}$, Alessio Celi ${ }^{4,5,6}$, Luca Dell'Anna ${ }^{7}$ and Andrea Trombettoni ${ }^{3,8}$ \\ 1 Dipartimento di Scienze Fisiche e Chimiche, Università dell'Aquila, via Vetoio, \\ I-67010 Coppito-L'Aquila, Italy \\ 2 INFN, Laboratori Nazionali del Gran Sasso, via G. Acitelli, 22, I-67100 Assergi (AQ), Italy \\ 3 SISSA, via Bonomea 265, I-34136 Trieste, Italy; anna.maraga@gmail.com (A.M.); andreatr@sissa.it (A.T.) \\ 4 Institute for Quantum Optics and Quantum Information, Austrian Academy of Sciences, A-6020 Innsbruck, \\ Austria; alessio.celi@gmail.com \\ 5 Institute for Quantum Optics and Quantum Information of the Austrian Academy of Sciences, \\ A-6020 Innsbruck, Austria \\ 6 ICFO-Institut de Ciencies Fotoniques, The Barcelona Institute of Science and Technology, \\ 08860 Castelldefels, Barcelona, Spain \\ 7 Dipartimento di Fisica e Astronomia “Galileo Galilei”, Università di Padova, via Marzolo 8, I-35131 Padova, \\ Italy; ldellann@gmail.com \\ 8 CNR-IOM DEMOCRITOS Simulation Center, via Bonomea 265, I-34136 Trieste, Italy \\ * Correspondence: llepori81@gmail.com
}

Received: 19 February 2018; Accepted: 12 April 2018; Published: 17 April 2018

\begin{abstract}
We show that a linear term coupling the atoms of an ultracold binary mixture provides a simple method to induce an effective and tunable population imbalance between them. This term is easily realized by Rabi coupling between different hyperfine levels of the same atomic species. The resulting effective imbalance holds for one-particle states dressed by the Rabi coupling and obtained by diagonalizing the mixing matrix of the Rabi term. This way of controlling the chemical potentials applies to both bosonic and fermionic atoms and it also allows for spatially- and temporally-dependent imbalances. As a first application, we show that, in the case of two attractive fermionic hyperfine levels with equal chemical potentials coupled by the Rabi pulse, the same superfluid properties of an imbalanced binary mixture are recovered. We finally discuss the properties of $m$-species mixtures in the presence of SU $(m)$-invariant interactions.
\end{abstract}

Keywords: quantum gases; multicomponent mixtures; rf-fields; Rabi coupling; imbalanced mixtures

\section{Introduction}

The average density of particles is a central parameter in ultracold experiments [1,2], and in general for condensed matter phenomena. Indeed, most physical systems acquire different features when the interaction energy is varied by changing the number of particles $N$. This fact arises already at the single-particle level, determining for instance the shape of the Fermi surfaces, in turn related, for example, to conductance or to the critical properties of Bose condensates [3]. The phenomenon is even more pronounced once interactions are included as, for instance, has been shown for superfluidity in fermion mixtures [4]. In the presence of a density imbalance between the pairing species, new types of superfluid phases, different from the standard BCS/BEC ones, can appear [4-12]. Other notable examples are the quantum Hall effect [13], where the filling deeply affects the nature of the ground state, or the transition superfluid-Mott insulator in the Bose-Hubbard model [2].

In recent years, the experimental capability of controlling and measuring local particle densities has greatly improved due to single-site addressing $[14,15]$. However, typically in ultracold experiments 
one controls the density and not the chemical potential $\mu$. In order to go from the canonical description to the grand-canonical one, one needs to exploit the relation between $N$ and $\mu$, generally a non-trivial task. Moreover, standard experiments with imbalanced mixtures are not always straightforward to realize, since often the phases separate or become unstable [2]. Therefore, designing alternative methods to tune imbalances effectively appears a very interesting task. This can be even more relevant if the chemical potential can be tuned in a space- or time-dependent way, which is not easy to realize controlling the atomic populations. For instance, this possibility may be crucial for the synthesis of unconventional superfluid pairings with nonzero momenta or for the creation of space-time defects.

In this paper, we provide a different method to induce and to control effective population imbalances in experiments involving atoms of a binary mixture linearly coupled between them, a major example being different hyperfine levels equally populated coupled by a Rabi rf-field. The obtained imbalances hold for dressed one-particle states, obtained diagonalizing the Rabi term that couples the hyperfine levels pairwise. The method works both for bosonic and fermionic atoms. The Rabi coupling in fermionic and bosonic mixtures is well studied for a variety of applications (see e.g., [16-21]) and widely used in ultracold atoms experiments [2,22,23]. We therefore think that it would be straightforward to perform an experiment along the lines discussed in this paper for realizing an effective imbalance between different hyperfine levels of ultracold mixtures.

In the presence of two-body interactions for the mixture, we argue that, under general conditions, the dynamics can be equally described in terms of the original states or of the dressed states, as the scattering processes do not destroy the coherence of the dressed states. A particularly interesting situation is when the two-body interactions do not depend on the involved hyperfine species in the mixture. This is the case of alkaline earth-like atoms, such as ${ }^{87} \mathrm{Sr}[24,25]$ or ${ }^{173} \mathrm{Yb}$ [26,27]. These atomic species provide instances of $\mathrm{SU}(m)$-invariant mixtures (with $m=10$ and 6, respectively). Quite recently, similar mixtures have been found important for the simulation of multispecies (anti-)ferromagnetism [28-31], synthetic dimensions and effective quantum Hall systems [32], and two-flavor symmetry locking [33]. We note also that SU(m) invariance may hold approximately also for atomic species such as ${ }^{87} \mathrm{Rb}$ or ${ }^{40} \mathrm{~K}[34,35]$.

The discussion above suggests that our method provides a new method for efficiently probing the physics of interacting imbalanced mixtures. As a first application, we focus on an attractive two-species fermionic mixture, showing that its superfluid properties under Rabi coupling are the same as those of an imbalanced mixture in the absence of a Rabi term. This example is particularly interesting in view of the rich phenomenology of the imbalanced Fermi gases as imbalance and interaction vary, also including FFLO physics (see e.g., [4,5] and references therein). Moreover, we argue that this mapping allows for engineering spatially- and temporally-dependent imbalances. They can be realized, for instance, by driving the intensity of the radio-frequency or of the Raman laser pulse which can induce Rabi oscillation, as recently done in a Floquet spin-orbit coupling experiment [36].

The paper is organized as follows. After a general survey on the proposed method (Section 2) in the presence of two-body interactions, we discuss the application of the Rabi coupling on an atomic mixture of two hyperfine species in a realistic experimental setup, and its generalization to $N$-species mixtures (Section 3). As a first example, we study a BCS/BEC superfluid in a mixture of two balanced hyperfine species coupled by a Rabi pulse (Section 4), finding agreement, even at the mean-field level, with the known literature on the two-species imbalanced fermionic superfluids in the absence of the Rabi coupling. In Section 5 we reconsider the same problem in the continuous space, discussing the effect of the Rabi coupling on the renormalization of the mean-field self-consistency equations for the nontrivial order parameters. In Section 6 we address further applications, involving the possibility of tuning the effective population imbalances in time and space, showing their experimental feasibility. Finally, in Section 7 we collect and discuss the main results, as well as possible future developments. 


\section{Two-Species Mixtures Coupled by Rabi Coupling}

In this section we describe a general lattice setup where a mixture of two bosonic or fermionic hyperfine species (labeled by $\sigma=\{\uparrow, \downarrow\}$ ) are coupled by a Rabi term. When not stated otherwise, these two species are supposed to have the same filling: $n_{\uparrow}=n_{\downarrow}$, with $n_{\sigma} \equiv \frac{N_{\sigma}}{V}, N_{\sigma}$ being the number of fermions of the species $\sigma$ on the lattice and $V$ the number of sites of the lattice.

A Rabi term can be induced by microwave or radio-frequency techniques, or by two-photon Raman transitions induced by two opportunely detuned laser beams [22,23]. The latter technique also allows for a spatial dependence of the Rabi frequency, as described in Section 6.

For simplicity, we will consider a three-dimensional cubic lattice, but, as discussed in the following, no significant changes arise in the continuum space or in lower dimensions. The atoms can hop between nearest neighbor sites with hopping energy $t(t>0)$ and interact via a contact attractive potential with magnitude $U$. The Rabi coupling flips the spin- $\sigma$ orientations of the atoms, with frequency $\Omega(t, \mathbf{r})$ which can be a complex value and may depend on time and position. We consider a real valued $\Omega(t, \mathbf{r})$ of a factorized form in time and space, $\Omega(t, \mathbf{r})=\Omega f(t) g(\mathbf{r})$. While by microwave or radio-frequency pulses a non-constant $g(\mathbf{r})$ is hard to obtain, by back-reflecting on a mirror the lasers inducing the Raman transitions, one can achieve a sinusoidal profile $g(\mathbf{r}) \propto \cos (\mathbf{r} \cdot q)$, where $\mathbf{q}$ is the difference between the wave-vectors of the two lasers.

In the discussion below we omit the space and time dependences, for which consequences will be discussed at the end of the paper, in Section 6.

\subsection{Fermions}

We focus first on fermions. Thus, the Hamiltonian describing the system that we are interested in is

$$
H=-t \sum_{<i, j>, \sigma} c_{i \sigma}^{\dagger} c_{j \sigma}+\Omega \sum_{i}\left(e^{i \varphi} c_{i \uparrow}^{\dagger} c_{i \downarrow}+e^{-i \varphi} c_{i \downarrow}^{\dagger} c_{i \uparrow}\right)-U \sum_{i} n_{i \uparrow} n_{i \downarrow}
$$

where $n_{i \sigma}=c_{i \sigma}^{\dagger} c_{i \sigma}$ are the number operators, and $\phi$ a generic phase.

Here we describe the system in the canonical ensemble, as for a single experimental realization, where the number of the atoms is fixed. The Hamiltonian (1) can be mapped by the unitary transformation

$$
a_{i \pm}=\frac{e^{-i \frac{\varphi}{2}} c_{i \uparrow} \pm e^{i \frac{\varphi}{2}} c_{i \downarrow}}{\sqrt{2}}
$$

to the following Hamiltonian

$$
H_{\text {ROT }}=-t \sum_{<i, j>, \alpha} a_{i \alpha}^{\dagger} a_{j \alpha}+\Omega \sum_{i}\left(n_{i+}-n_{i-}\right)-U \sum_{i} n_{i+} n_{i-},
$$

where $\alpha= \pm$ and $n_{i \pm}=a_{i \pm}^{\dagger} a_{i \pm}$. We find that the original Rabi coupling $\propto \Omega$ is mapped to an energy imbalance $h=2 \Omega$, as for a Zeeman term. Even more importantly, the interaction $\propto U$ transforms covariantly under the same transformation [37], allowing us to probe the Hubbard physics on imbalanced Hamiltonians also (an example will be given subsequently). We finally mention that, due to the Rabi coupling, an imbalance at $\Omega=0$ does not produce any qualitative difference, as compared to the balanced case, once $\Omega$ is switched on (see e.g., [38]).

The Zeeman term can be also interpreted as a chemical potential term. Indeed, as we will describe in Section 4, although the total number of particles $N_{+}+N_{-}=N_{\uparrow}+N_{\downarrow} \equiv N$ remains constant in the present canonical scheme, the number $N_{ \pm}$is not fixed but can fluctuate, and therefore $N_{ \pm}$can be considered as quantum averages. In particular, we will discuss the behaviour of the average difference $\left(N_{+}-N_{-}\right)$as a function of $U$ and $\Omega$, non vanishing in a normal state.

The calculations above are also valid in the grand canonical ensemble, introducing a chemical potential $\mu$ and then letting $N_{\uparrow}\left(n_{i, \uparrow}\right)$ and $N_{\downarrow}\left(n_{i, \downarrow}\right)$ fluctuate, which is then fixed only in the average. 
In real experiments the $\mu$-term in (4) can be ascribed to the average occupation numbers for different experimental realizations. The resulting Hamiltonian reads:

$$
H=-t \sum_{<i, j>, \sigma} c_{i \sigma}^{\dagger} c_{j \sigma}+\Omega \sum_{i}\left(c_{i \uparrow}^{\dagger} c_{i \downarrow}+c_{i \downarrow}^{\dagger} c_{i \uparrow}\right)-U \sum_{i} n_{i \uparrow} n_{i \downarrow}-\mu \sum_{i, \sigma} c_{i \sigma}^{\dagger} c_{i \sigma}
$$

and the transformation (2) yields:

$$
\begin{array}{r}
H_{\text {ROT }}=-t \sum_{<i, j>, \alpha} a_{i \alpha}^{\dagger} a_{j \alpha}-(\mu-\Omega) \sum_{i} n_{i+}-(\mu+\Omega) \sum_{i} n_{i-}-U \sum_{i} n_{i+} n_{i-} \\
=-t \sum_{<i, j>, \alpha} a_{i \alpha}^{\dagger} a_{j \alpha}-\mu \sum_{i}\left(n_{i+}+n_{i-}\right)+\Omega \sum_{i}\left(n_{i+}-n_{i-}\right)-U \sum_{i} n_{i+} n_{i-} .
\end{array}
$$

We find that the Rabi term in the old basis $\{\uparrow, \downarrow\}$ becomes, after the transformation in (2), an imbalance term $\delta \mu=2 \Omega$ for the chemical potentials of the new species $a_{ \pm}$.

\subsection{Bosons}

For a bosonic system, three Hubbard interactions are possible: the intra-species interactions $U_{\uparrow \uparrow}$ and $U_{\downarrow \downarrow}$, and the inter-species interaction $U_{\uparrow \downarrow}$, so that we get

$$
H=-t \sum_{<i, j>, \sigma} c_{i \sigma}^{\dagger} c_{j \sigma}+\Omega \sum_{i}\left(e^{i \varphi} c_{i \uparrow}^{\dagger} c_{i \downarrow}+e^{-i \varphi} c_{i \downarrow}^{\dagger} c_{i \uparrow}\right)-\sum_{i}\left(U_{\uparrow \uparrow} n_{i \uparrow}^{2}+U_{\downarrow \downarrow} n_{i \downarrow}^{2}+U_{\uparrow \downarrow} n_{i \uparrow} n_{i \downarrow}\right) .
$$

The interacting term of this Hamiltonian transforms covariantly under the rotation in Equation (2) iff $U_{\uparrow \uparrow}=U_{\downarrow \downarrow}=U_{\uparrow \downarrow} / 2$, which is the case for alkaline earth atoms [28,29]. In this case, with these relations for the interaction strengths, applying Equation (2), Equation (6) maps to

$$
H_{\mathrm{ROT}}=-t \sum_{<i, j>, \alpha} a_{i \alpha}^{\dagger} a_{j \alpha}+\Omega \sum_{i}\left(n_{i+}-n_{i-}\right)-\frac{U_{\uparrow \downarrow}}{2} \sum_{i}\left(n_{i+}+n_{i-}\right)^{2} .
$$

\subsection{Trap Effects}

In current ultracold atoms experiments, both for bosons and fermions, a typical ingredient is the external trapping potential of the form

$$
\sum_{i} V\left(\mathbf{r}_{i}\right)\left(n_{i \uparrow}+n_{i \downarrow}\right),
$$

where $V\left(\mathbf{r}_{i}\right)=\frac{m}{2} \omega^{2}\left|\mathbf{r}_{i}\right|^{2}$ and $\mathbf{r}_{i}$ is the vector distance of the lattice site at position $i$ from the center of the trap. We assume the trapping frequency $\omega$ and the mass $m$ equal for both the species $\{\uparrow, \downarrow\}$, as in most of the experiments involving different hyperfine levels of the same atom. It is straightforward to show that also the potential in (8) transforms covariantly under the rotation in Equation (2):

$$
\sum_{i} V\left(\mathbf{r}_{i}\right)\left(n_{i+}+n_{i-}\right),
$$

and then all the previous discussions are not spoiled by the presence of this term.

In the light of the above consideration, we expect that the properties of balanced mixtures (both in the canonical and in the grandcanonical ensemble) under the Rabi coupling and possibly of two-body interactions (provided that the interactions do not to spoil the rotated states (2)) are equal to the physics of imbalanced interacting mixtures. This will be further studied in Section 4, considering the superfluid properties of the Hamiltonian (Equation (3)). 


\subsection{Comments on the Experimental Implementation}

In this subsection, we comment on the limits of tunability of the Rabi coupling, also in relation to the other parameters, such as the typical hopping amplitude $\{t\}$ and the strengths of the interaction $\left\{U_{i}\right\}$. Indeed our method, to be effective, requires the Rabi coupling to be tunable over a range of energies at least comparable with the smallest energy scales between the hopping amplitude and the interactions. This condition is easily fulfilled for constant (or smoothly varying) $\Omega$. Indeed, it is possible to achieve $\Omega \sim t$ within the validity of the tight-binding approximation. This favourable situation can change if the Rabi frequency depends on the position or on the time. Roughly speaking, the allowed dependence should fulfill the adiabatic theorem. In Section 6.2, we quantitatively discuss the limits imposed by the presence of a sinusoidal behavior for $\Omega$ induced by Raman lasers. In particular we will show that the related momentum transfer does not considerably limit further the range of allowed values for $\Omega$. Thus, it turns out that there are neither conceptual nor technical limitations in the use of the Rabi coupling technique. Nowadays intensities $\Omega \sim \mathrm{kHz}$ are realistic, which are of the same order of magnitude of the Fermi energies in typical experiments, both in the continuous space and on the lattice. We conclude that unbalancing an attractive two-species hyperfine mixture by Rabi coupling allows us to investigate (at least) both the BCS and BEC limits of the superfluid regime for ultracold fermionic mixtures.

\subsection{Further Generalizations}

In principle, spin-dependent hopping $t_{\sigma}$ can be added to (4), for instance exploiting superlattice configurations (see, e.g., [2] and references therein). Interestingly, if we apply the transformation in (2) to the resulting Hamiltonian, we obtain:

$$
\begin{aligned}
H_{t, \mathrm{ROT}}= & -\frac{t_{\uparrow}+t_{\downarrow}}{2} \sum_{<i, j>, \alpha} a_{i \alpha}^{\dagger} a_{j \alpha}-\frac{t_{\uparrow}-t_{\downarrow}}{2} \sum_{<i, j>}\left(a_{i+}^{\dagger} a_{j-}+a_{i-}^{\dagger} a_{j+}\right) \\
& -(\mu-\Omega) \sum_{i} n_{i+}-(\mu+\Omega) \sum_{i} n_{i-}-U \sum_{i} n_{i+} n_{i-},
\end{aligned}
$$

We find that, in the rotated frame, in addition to the spin-dependent chemical potential, a spin-orbit-like coupling term appears. Therefore, this scheme can be used to simulate a spin-orbit coupling, at least in one-dimensional lattices. If we proceed further with the diagonalization, we end up with the Hamiltonian

$$
H_{t, \mathrm{ROT}}^{(\mathrm{diag})}=\sum_{\mathbf{k}}\left[\lambda_{+}(\mathbf{k}) a_{\mathbf{k}+}^{\dagger} a_{\mathbf{k}+}+\lambda_{-}(\mathbf{k}) a_{\mathbf{k}-}^{\dagger} a_{\mathbf{k}-}-U n_{\mathbf{k}+} n_{\mathbf{k}-}\right]
$$

with

$$
\lambda_{ \pm}(k)=\varepsilon_{\mathbf{k}}-\mu \pm \sqrt{\delta \varepsilon_{\mathbf{k}}^{2}+\Omega^{2}}
$$

where $\varepsilon_{\mathbf{k}} \equiv \frac{\varepsilon_{\mathbf{k} \uparrow}+\epsilon_{\mathbf{k} \downarrow}}{2}$ and $\delta \varepsilon_{\mathbf{k}} \equiv \frac{\varepsilon_{\mathbf{k} \uparrow}-\varepsilon_{\mathbf{k} \downarrow}}{2}$, posing $\varepsilon_{\mathbf{k} \uparrow}=-t_{\uparrow} \sum_{s=x, y, z} \cos k_{s}, \varepsilon_{\mathbf{k} \downarrow}=-t_{\downarrow} \sum_{s=x, y, z} \cos k_{s}$.

\section{Generalization to $N$ Species}

The model described in the previous section can be easily extended to a $N$-mixture $(N=2 M)$ of bosonic or fermionic atoms in several ways, for instance by coupling the various hyperfine species pairwise. The non-interacting grand canonical Hamiltonian with possible imbalances in the densities (with different chemical potentials) and in the hopping amplitudes reads in this case:

$$
\begin{aligned}
H_{\left\{\mu_{l}\right\},\left\{t_{l}\right\}=} & -\sum_{<i, j>, l} t_{2 l} c_{i, 2 l}^{\dagger} c_{j, 2 l}-\sum_{<i, j>, l} t_{2 l+1} c_{i, 2 l+1}^{\dagger} c_{j, 2 l+1} \\
& -\sum_{i, l}\left(\mu_{2 l} c_{i 2 l}^{\dagger} c_{i, 2 l}+\mu_{2 l+1} c_{i 2 l+1}^{\dagger} c_{i, 2 l+1}\right)+\sum_{i, l} \Omega_{l}\left(c_{i, 2 l}^{\dagger} c_{i, 2 l+1}+c_{i, 2 l+1}^{\dagger} c_{i, 2 l}\right),
\end{aligned}
$$


where $l=1, \ldots, M$ labels the pairs of hyperfine species coupled by the Rabi coupling.

This Hamiltonian, after the unitary transformation

$$
a_{i \pm}^{(l)}=\frac{c_{i, 2 l} \pm c_{i, 2 l+1}}{\sqrt{2}}
$$

becomes

$$
\begin{aligned}
& H_{\left\{\mu_{l}\right\},\left\{t_{l}\right\} \text { ROT }}=-\sum_{<i, j>, \alpha, l} \frac{t_{2 l}+t_{2 l+1}}{2} a_{i \alpha}^{(l) \dagger} a_{j \alpha}^{(l)}-\sum_{<i, j>, l} \frac{t_{2 l}-t_{2 l+1}}{2}\left(a_{i+}^{(l) \dagger} a_{j-}^{(l)}+a_{i-}^{(l) \dagger} a_{j+}^{(l)}\right) \\
& -\sum_{i, l}\left[\left(\frac{\mu_{2 l}+\mu_{2 l+1}}{2}-\Omega_{l}\right) n_{i+}^{(l)}+\left(\frac{\mu_{2 l}+\mu_{2 l+1}}{2}+\Omega_{l}\right) n_{i-}^{(l)}\right]-\sum_{i, l} \frac{\mu_{2 l}-\mu_{2 l+1}}{2}\left(a_{i+}^{(l) \dagger} a_{i-}^{(l)}+a_{i-}^{(l) \dagger} a_{i+}^{(l)}\right) .
\end{aligned}
$$

Possible density-density interactions have the same form also in the dressed basis (14), provided that the interactions involve only the hyperfine species pairwise $2 l-(2 l+1)$ :

$$
\sum_{l=1}^{M} \sum_{i, j} V_{l}(i-j)\left(n_{i, 2 l}+n_{i, 2 l+1}\right)\left(n_{j, 2 l}+n_{j, 2 l+1}\right)
$$

$\left(n_{i, \alpha}=c_{i, \alpha}^{\dagger} c_{i, \alpha}\right)$

In this case, the transformation (14) yields

$$
\sum_{l=1}^{M} \sum_{i, j} V_{l}(i-j)\left(n_{i+}^{(l)}+n_{i-}^{(l)}\right)\left(n_{j+}^{(l)}+n_{j-}^{(l)}\right),
$$

where $n_{i \pm}^{(l)}=a_{i \pm}^{\dagger(l)} a_{i \pm}^{(l)}$.

Another case, even more interesting from the experimental point of view, occurs when the interactions pair all the hyperfine species in the mixtures, with the same strength and the same space dependence (as for alkaline earth-like atoms [28-30,32], see also the Introduction):

$$
\sum_{i, j} V(i-j) \sum_{s, s^{\prime}=1}^{2 M} n_{i, s} n_{j, s^{\prime}}=\sum_{i, j} V(i-j)\left(\sum_{s=1}^{2 M} n_{i, s}\right)\left(\sum_{s^{\prime}=1}^{2 M} n_{j, s^{\prime}}\right) .
$$

This interaction still transforms covariantly under (14):

$$
\sum_{i, j} V(i-j) \sum_{l, l^{\prime}=1}^{M}\left(n_{i+}^{(l)}+n_{i-}^{(l)}\right)\left(n_{j+}^{\left(l^{\prime}\right)}+n_{j-}^{\left(l^{\prime}\right)}\right) .
$$

In these cases, the dynamics can be described easily in terms of dressed states as well, since the scattering processes related to the interactions do not spoil them.

A clarification of the latter point can be obtained considering the example of two species, $\uparrow$ and $\downarrow$ (labeled by the momentum), scattering in the s-wave channel. Due to the Pauli principle, the low-energy scattering, supposedly elastic, can occur only in the channel

$$
\frac{1}{\sqrt{2}}\left[\frac{\uparrow+\downarrow}{\sqrt{2}} \otimes \frac{\uparrow-\downarrow}{\sqrt{2}}-\frac{\uparrow-\downarrow}{\sqrt{2}} \otimes \frac{\uparrow+\downarrow}{\sqrt{2}}\right](\mathbf{k})=\frac{\uparrow \downarrow-\downarrow \uparrow}{\sqrt{2}}(\mathbf{k}) \rightarrow e^{i \phi_{\mathbf{k}}} \frac{\uparrow \downarrow-\downarrow \uparrow}{\sqrt{2}}(\mathbf{k}),
$$

with $\phi_{\mathbf{k}}$ being the momentum depending scattering phase. We find that $e^{i \phi_{\mathbf{k}}}$ globally multiplies the final scattering state, without spoiling the relative coherence of the states $\uparrow \downarrow$ and $\downarrow \uparrow$. This is an effect of the linearity of the scattering matrix [39] and it immediately holds if $N=2$. The phase $e^{i \phi_{\mathbf{k}}}$ is nothing but the phase resulting from the scattering at $\Omega=0$ of $\uparrow$ and $\downarrow$ particles, with momentum $\mathbf{k}$ in the spin singlet state. 
In the general case, with $N \geq 2$, the conditions that we imposed above on the interactions assure that every scattering process changes the initial state $\frac{|l\rangle \pm|l+1\rangle}{\sqrt{2}}$ just by multiplying it by a pure phase $e^{i \phi_{l k}}$, as for the case reported in Equation (20).

\section{Two-Species Superfluidity in the Presence of Rabi Coupling}

In this section, we exemplify the effect of an imbalance induced by Rabi coupling, focusing on a two-species fermionic mixture and studying the properties of its superfluid phase. We consider the Hamiltonian in (4):

$$
H=-t \sum_{<i, j>, \sigma} c_{i \sigma}^{\dagger} c_{j \sigma}-\mu \sum_{i, \sigma} c_{i \sigma}^{\dagger} c_{i \sigma}+\Omega \sum_{i}\left(c_{i \uparrow}^{\dagger} c_{i \downarrow}+c_{i \downarrow}^{\dagger} c_{i \uparrow}\right)-U \sum_{i} n_{i \uparrow} n_{i \downarrow} .
$$

As discussed in Section 2, we explicitly include the chemical potential term. In the following, this Hamiltonian and similar ones with an Hubbard interaction will be denoted as "full" Hamiltonians, in contrast to the mean field quadratic Hamiltonians.

In the presence of a nonzero superfluid gap $\Delta$ (to be verified a posteriori), the corresponding (mean field) BCS-projected Hamiltonian is:

$$
H=-t \sum_{<i, j>, \sigma} c_{i \sigma}^{\dagger} c_{j \sigma}-\left(\mu+\frac{U n}{2}\right) \sum_{i, \sigma} c_{i \sigma}^{\dagger} c_{i \sigma}+(\Omega+\gamma) \sum_{i}\left(c_{i \uparrow}^{\dagger} c_{i \downarrow}+c_{i \downarrow}^{\dagger} c_{i \uparrow}\right)-\Delta \sum_{i}\left(c_{i \uparrow}^{\dagger} c_{i \downarrow}^{\dagger}+c_{i \downarrow} c_{i \uparrow}\right) .
$$

In Equation (22) we assumed the presence of a further bilinear order parameter, also coming from the Wick decomposition of the Hubbard interaction term in Equation (21):

$$
\gamma=-U\left\langle c_{i \uparrow}^{\dagger} c_{i \downarrow}\right\rangle=-U\left\langle c^{\dagger}{ }_{i \downarrow} c_{i \uparrow}\right\rangle,
$$

due to the presence of the Rabi coupling. Moreover, we fix $(\Omega+\gamma)>0$, up to a phase redefinition of the $c_{i \sigma}$ operators. Going to momentum space, the Hamiltonian (22) can be easily diagonalized, finding the eigenvalues:

$$
\lambda_{\mathbf{k}}^{( \pm)}=E_{\mathbf{k}} \pm(\Omega+\gamma),
$$

where $E_{\mathbf{k}}=\sqrt{\xi_{\mathbf{k}}^{2}+\Delta^{2}}$ and

$$
\xi_{\mathbf{k}}=\varepsilon_{\mathbf{k}}-\tilde{\mu}, \quad \varepsilon_{\mathbf{k}}=-2 t \sum_{l=x, y, z} \cos k_{l}, \quad \tilde{\mu}=\mu+\frac{U N}{2 V} .
$$

The Bogoliubov coefficients turn out to be the same as in the purely $(\Omega=0)$ BCS case. However, we find that, introducing the Rabi term, the quasiparticle excitation spectrum is split into two bands, shifted by $\pm(\Omega+\gamma)$ with respect to the usual BCS case. Correspondingly, the minimum of the excitation spectrum is

$$
\Delta_{G}=\Delta-|\Omega+\gamma|
$$

The ground-state energy reads:

$$
E_{\text {SUP }}=\frac{V \Delta^{2}}{U}+\frac{1}{2} \sum_{\mathbf{k}}\left[\left(\xi_{\mathbf{k}}-\lambda^{(+)}(\mathbf{k})\right)+\left(\xi_{\mathbf{k}}-\lambda^{(-)}(\mathbf{k})\right)\right]+\sum_{\mathbf{k} \in \overline{\mathcal{D}}} \lambda^{(-)}(\mathbf{k}),
$$

where $\mathcal{D}$ is a domain in the first Brillouin zone defined as

$$
\mathcal{D}=\left\{\mathbf{k} \in 1^{\mathrm{st}} \mathrm{BZ}: E_{\mathbf{k}}>\Omega+\gamma\right\}
$$


and its complementary domain as

$$
\overline{\mathcal{D}}=\left\{\mathbf{k} \in 1^{\mathrm{st}} \mathrm{BZ}: E_{\mathbf{k}}<\Omega+\gamma\right\} .
$$

The explicit calculation of the self-consistent equations for the order parameters $\Delta, \gamma$, and $\mu$ is performed by setting to 0 the derivatives of $E_{\mathrm{SUP}}+\mu N+\gamma \delta N, \delta N \equiv\left(N_{-}-N_{+}\right)$, with regards to $\Delta$, $\gamma$ and $\mu$, respectively.

The introduction of the quantity $\gamma \delta N$ stems from the Wick decomposition of the Hubbard interaction in (21), similar to the first term in $E_{\mathrm{SUP}}$ [40]. The final result is:

$$
\begin{aligned}
& 1=\frac{U}{2 V} \sum_{\mathbf{k} \in \mathcal{D}} \frac{1}{E_{\mathbf{k}}}, \\
& \delta N=-\sum_{\mathbf{k} \in \overline{\mathcal{D}}} 1, \\
& n=\frac{1}{V} \sum_{\mathbf{k}}\left(1-\frac{\xi_{\mathbf{k}}}{E_{\mathbf{k}}}\right)+\frac{1}{V} \sum_{\mathbf{k} \in \mathcal{D}} \frac{\xi_{\mathbf{k}}}{E_{\mathbf{k}}}=1-\frac{1}{V} \sum_{\mathbf{k} \in \mathcal{D}} \frac{\xi_{\mathbf{k}}}{E_{\mathbf{k}}} .
\end{aligned}
$$

From (31), we conclude that $\gamma \leq 0$, with $\gamma=0$ (as well as $\overline{\mathcal{D}}=0$ ) iff $\Omega=0$; moreover in (32) an additional term is present, compared to the $\Omega=0$ case.

We observe that the domain $\mathcal{D}$, as well as its complementary $\overline{\mathcal{D}}$, differs from the definition given implicitly in [5], where the contribution of the imbalance $\delta N$ has been neglected in the Hartree terms $-U N_{-} \sum_{i} a_{i+}^{+} a_{i+}-U N_{+} \sum_{i} a_{i-}^{\dagger} a_{i-}$. In this case, it holds that $\mathcal{D}=\left\{\mathbf{k} \in 1^{\text {st }} \mathrm{BZ}: E_{\mathbf{k}}>\Omega+\gamma\right\}$ and Equation (31) is simplified. This aspect will be discussed in Section 5, where we will infer that, if $U=0$, the parameter $\delta N$ equals the imbalance of two fermionic species with chemical potentials $\mu_{ \pm} \equiv \mu \pm \Omega$. The same identification holds for the ground-state energy (27).

\subsection{Comparison at Mean-Field Level}

The mean field ground-state energy (27), resulting from the full Hamiltonian (21), equals the same quantity for a imbalanced mixture under an onsite attraction [5], as described by the Hamiltonian (5). Indeed, we found that the Hamiltonian (21) maps exactly to (5) under the transformation in (2); for this reason the spectra and the phenomenologies at these two full Hamiltonians must be the same. However, one can ask whether the equivalence exactly established is valid also at mean-field level.

In the following we show, as may be expected, that the equivalence is valid as well for the corresponding Hamiltonians obtained in mean-field approximations, i.e., that the previous result holds at the mean-field level (where the self-consistency conditions have to be enforced).

The mean-field Hamiltonian from (5) reads:

$$
\begin{aligned}
H_{\mathrm{ROT}}=-t \sum_{<i, j>, \alpha} & a_{i \alpha}^{\dagger} a_{j \alpha}-(\mu-\Omega) \sum_{i} a_{i+}^{\dagger} a_{i+}-(\mu+\Omega) \sum_{i} a_{i-}^{\dagger} a_{i-} \\
& -U N_{-} \sum_{i} a_{i+}^{\dagger} a_{i+}-U N_{+} \sum_{i} a_{i-}^{\dagger} a_{i-}+\Delta \sum_{i}\left(a_{i+} a_{i-}+\text { h.c. }\right)+\mu N+\Theta \delta N,
\end{aligned}
$$

Similar to the previous subsection, the self-consistent equations for $\Delta, N, \delta N$ can be found deriving $E_{\mathrm{SUP}}+\mu N+\Omega \delta N$ with respect to $\Delta, \Theta$, and $\mu$ [5]. The results are the same as in Equations (30)-(32).

We can check at this point the mean-field Hamiltonian obtained from (22) by the transformation in (2)

$$
\begin{aligned}
H_{\text {ROT }}=-t \sum_{<i, j>, \alpha} a_{i \alpha}^{\dagger} a_{j \alpha}-(\mu-\Omega-\gamma) \sum_{i} a_{i+}^{\dagger} a_{i+}-(\mu+\Omega+\gamma) \sum_{i} a_{i-}^{\dagger} a_{i-} \\
-\frac{U}{2} N \sum_{i}\left(a_{i+}^{\dagger} a_{i+}+a_{i-}^{\dagger} a_{i-}\right)+\Delta \sum_{i}\left(a_{i+} a_{i-}+\text { h.c. }\right)+\mu N+(\Omega+\gamma) \delta N,
\end{aligned}
$$


coincides with the mean-field Hamiltonian (33). Interestingly, this comparison sheds light on the physical meaning of the parameter $\gamma$, yielding indeed:

$$
\gamma=\frac{U}{2} \delta N
$$

Summing up, we found that the relation in (35) for the mean-field parameter $\gamma$ is imposed by the request of equality between the mean-field Hamiltonians in (33) and (34), a fact physically motivated, but not trivially implied, by the equivalence of the full Hamiltonians in (21) and (5).

\subsection{Disscusions}

Since the Hamiltonians in Equations (5) and (33) have been studied extensively in various papers devoted to imbalanced fermionic mixtures $[4,41]$, both at zero and at finite temperatures, we do not further study their properties, and refer to the pertinent literature. For our purposes, indeed, the main point we want to stress here is the equivalence of these Hamiltonians in the presence of Rabi couplings with those describing imbalanced Fermi mixtures. In the experiments, one performs measurements on the fermionic species $c^{\prime}$ s; from the obtained findings for the quantities $\left\langle c^{\dagger} c\right\rangle$ and $\left\langle c^{\dagger} c^{\dagger}\right\rangle$, and via the relation between the $c^{\prime}$ s and the $a^{\prime}$ s, one can then reconstruct the phase diagram of the (a-)imbalanced mixture. The resulting main feature, based on the available results, is the appearance, both on the BCS and BEC sides, of a coexistence of normal and superfluid phases for suitable effective imbalances $\delta \mu=2 \Omega$. Moreover, unconventional forms of superconductivity, such as FFLO, are conjectured (for an extended review, see [6]). By the definition of $\gamma$, we expect that $\gamma=0$ in a superfluid phase, where $N_{+}=N_{-}$.

\section{The Continuous Case}

In this section we deal with the analogous equation (1) in the continuum space. In this situation, most of the features are qualitatively equal to the lattice case, and then we detail only the formulation and discussion of the equations for $\gamma, \mu$, and $\Delta$. In particular, we discuss the removal of the infinities encountered during their solution. We again assume work in three dimensions. The Hamiltonian is:

$$
\begin{aligned}
H^{\text {(cont) }=}= & \frac{1}{V} \sum_{\sigma} \int \mathrm{d} \mathbf{r} c_{\sigma}^{\dagger}(\mathbf{r})\left(-\hbar^{2} \frac{\nabla^{2}}{2 m}-\mu\right) c_{\sigma}(\mathbf{r}) \\
& +\frac{\Omega}{V} \int \mathrm{d} \mathbf{r}\left(c_{\uparrow}^{\dagger}(\mathbf{r}) c_{\downarrow}(\mathbf{r})+c_{\downarrow}^{\dagger}(\mathbf{r}) c_{\uparrow}(\mathbf{r})\right)-\frac{U}{V} \int \mathrm{d} \mathbf{r} n_{\uparrow}(\mathbf{r}) n_{\downarrow}(\mathbf{r}),
\end{aligned}
$$

with $V$ denoting in this section the volume. The eigenstates of (36) in the absence of the interaction term $H_{i}=-\frac{U}{V} \int \mathrm{d} \mathbf{r} n_{\uparrow}(\mathbf{r}) n_{\downarrow}(\mathbf{r})$ are superpositions of plane waves with momentum $\mathbf{k}$, of the form $a_{ \pm}(\mathbf{k})=\frac{c_{\uparrow} \pm c_{\downarrow}}{\sqrt{2}}(\mathbf{k})$, as in (2). These states are assumed to be asymptotical in the scattering evolution [34], and the interaction to arise between them without coherence-spoiling effects. Since here $N=2$ in the notation of Section 3, this requirement is automatically fulfilled (see the discussion therein). The Hamiltonian transformed by (2) reads:

$$
\begin{aligned}
H_{\mathrm{ROT}}^{(\text {cont })=} & -\frac{\hbar^{2}}{V} \sum_{\alpha= \pm} \int \mathrm{d} \mathbf{r} a_{\alpha}^{\dagger}(\mathbf{r}) \frac{\nabla^{2}}{2 m} a_{\alpha}(\mathbf{r})-\frac{(\mu-\Omega)}{V} \int \mathrm{d} \mathbf{r} a_{+}^{\dagger}(\mathbf{r}) a_{+}(\mathbf{r}) \\
& -\frac{(\mu+\Omega)}{V} a_{-}^{\dagger}(\mathbf{r}) a_{-}(\mathbf{r})-\frac{U}{V} \int \mathrm{d} \mathbf{r} n_{+}(\mathbf{r}) n_{-}(\mathbf{r}) .
\end{aligned}
$$




\section{Gap Equations}

We consider the zero-temperature self-consistency equations for $\gamma, \mu$, and $\Delta$. These equations have the same functional form as in the lattice case:

$$
\begin{aligned}
& 1=\frac{U}{2} \frac{V}{(2 \pi \hbar)^{3}} \int_{\mathcal{D}} \mathrm{d} \mathbf{k} \frac{1}{E_{\mathbf{k}}} \\
& \gamma=-\frac{U}{2} \frac{V}{(2 \pi \hbar)^{3}} \int_{\overline{\mathcal{D}}} \mathrm{d} \mathbf{k} 1 \\
& n=1-\frac{1}{(2 \pi \hbar)^{3}} \int_{\mathcal{D}} \mathrm{d} \mathbf{k} \frac{\xi_{\mathbf{k}}}{E_{\mathbf{k}}},
\end{aligned}
$$

similarly, the domains $\mathcal{D}$ and $\overline{\mathcal{D}}$ are the same as in Section 4 . Indeed, no assumption has been made about the precise form of $\varepsilon_{\mathbf{k}}$ in the derivation of the equations for $\gamma, \mu$, and $\Delta$. Clearly, $\mathcal{D}$ and $\overline{\mathcal{D}}$ now take values into the infinite set of all the possible three-dimensional momenta, and not any longer in the first Brillouin zone. In order to derive (38)-(40) we also used the fact that $\sum_{\mathbf{k}} \rightarrow \frac{V}{(2 \pi \hbar)^{3}} \int \mathrm{d} \mathbf{k}$, passing to the continuum limit.

In the absence of Rabi coupling, Equation (38) is known to be divergent and need regularization by the introduction of the scattering lengths $a$ [4]. Let us study what happens to (38)-(40) in the presence of a Rabi term. To do this, it is useful to reconsider their derivation in the presence of a finite range potential $U(\mathbf{r})$, generalizing the equation in Section 4 (where a $\delta(\mathbf{r})$ interaction has been assumed instead). The result is:

$$
\begin{aligned}
& \Delta_{\mathbf{k}^{\prime}}=\frac{1}{2} \frac{1}{(2 \pi \hbar)^{3}} \int_{\mathcal{D}} \mathrm{d} \mathbf{k} \frac{\tilde{U}\left(\mathbf{k}, \mathbf{k}^{\prime}\right)}{\sqrt{\xi_{\mathbf{k}}^{2}+\Delta_{\mathbf{k}}^{2}}} \Delta_{\mathbf{k}}, \\
& \gamma_{\mathbf{k}^{\prime}}=-\frac{1}{2} \frac{1}{(2 \pi \hbar)^{3}} \int_{\overline{\mathcal{D}}} \mathrm{d} \mathbf{k} \tilde{U}\left(\mathbf{k}, \mathbf{k}^{\prime}\right) \\
& n=1-\frac{1}{(2 \pi \hbar)^{3}} \int_{\mathcal{D}} \mathrm{d} \mathbf{k} \frac{\xi_{\mathbf{k}}}{E_{\mathbf{k}}}
\end{aligned}
$$

where $\tilde{U}\left(\mathbf{k}, \mathbf{k}^{\prime}\right)=\tilde{U}\left(\mathbf{k}-\mathbf{k}^{\prime}\right)$ denotes the Fourier transform of $U(\mathbf{r}), \tilde{U}(\mathbf{q})=\int \mathrm{d} \mathbf{r} e^{i \mathbf{q} \cdot \mathbf{r}} U(\mathbf{r})$. We insert into these equations the scattering amplitude (for the moment not restricted to the $l=0$ contribution) $f\left(\mathbf{k}, \mathbf{k}^{\prime}\right)$, via the formula [39,42]:

$$
f\left(\mathbf{k}, \mathbf{k}^{\prime}\right)=\frac{m}{4 \pi \hbar^{2}} \tilde{U}\left(\mathbf{k}, \mathbf{k}^{\prime}\right)+\frac{1}{(2 \pi \hbar)^{3}} \int \mathrm{d} \mathbf{q} \frac{\tilde{U}\left(\mathbf{k}^{\prime}, \mathbf{q}\right) f(\mathbf{k}, \mathbf{q})}{\left(\varepsilon_{\mathbf{k}}-\varepsilon_{\mathbf{q}}-i 0^{+}\right)} .
$$

The solution of this equation can be obtained by iteration on $f\left(\mathbf{p}, \mathbf{p}^{\prime}\right)[39]$ in the second term to the right side:

$$
f\left(\mathbf{k}, \mathbf{k}^{\prime}\right)=\frac{m}{4 \pi \hbar^{2}}\left[\tilde{U}\left(\mathbf{k}, \mathbf{k}^{\prime}\right)+\frac{1}{(2 \pi \hbar)^{3}} \int \mathrm{d} \mathbf{q} \frac{\tilde{U}\left(\mathbf{k}^{\prime}, \mathbf{q}\right) \tilde{U}(\mathbf{k}, \mathbf{q})}{\left(\varepsilon_{\mathbf{k}}-\varepsilon_{\mathbf{q}}-i 0^{+}\right)}+\ldots\right]
$$

the approximation keeping only the first term of (45) quoted as the (first) Born approximation [39]. Multiplying now both of the terms in (44) for $\mathbf{d} \mathbf{k}^{\prime}$, performing this integration, and exploiting (44), we obtain:

$$
\Delta_{\mathbf{k}^{\prime}}=\frac{1}{2} \frac{4 \pi \hbar^{2}}{m} \frac{1}{(2 \pi \hbar)^{3}}\left(\int_{\mathcal{D}} \mathrm{d} \mathbf{k} \frac{f\left(\mathbf{k}, \mathbf{k}^{\prime}\right)}{\sqrt{\xi_{\mathbf{k}}^{2}+\Delta_{\mathbf{k}}^{2}}}-\int \mathrm{d} \mathbf{k} \frac{f\left(\mathbf{k}, \mathbf{k}^{\prime}\right)}{\varepsilon_{\mathbf{k}}-\varepsilon_{\mathbf{k}^{\prime}}}\right) \Delta_{\mathbf{k}} .
$$


Let us restrict now the scattering in the low-energy limit, where $\mathbf{k}, \mathbf{k}^{\prime} \rightarrow 0$ : in this regime $\Delta_{\mathbf{k}, \mathbf{k}^{\prime}} \rightarrow \Delta$ and $f\left(\mathbf{k}, \mathbf{k}^{\prime}\right) \rightarrow-a$ ( $a$ being the scattering length in s-wave, supposed negative) [39], so that we obtain:

$$
1=-\frac{1}{2} \frac{4 \pi \hbar^{2} a}{m} \frac{1}{(2 \pi \hbar)^{3}}\left(\int_{\mathcal{D}} \mathrm{d} \mathbf{k} \frac{1}{\sqrt{\xi_{\mathbf{k}}^{2}+\Delta_{\mathbf{k}}^{2}}}-\int \mathrm{d} \mathbf{k} \frac{1}{\varepsilon_{\mathbf{k}}}\right) .
$$

This equation is the renormalized version of (38). Note that the integration on the second term in the right part of (47) is onto all the possible momenta, independent on the range of integration $\mathcal{D}$ of the first term, as in the absence of the Rabi term. The result is expected in the light of the mapping of (4) on (5): the effective imbalance in the last Hamiltonian does not affect the interaction.

We deal now with (42): this is finite since $\overline{\mathcal{D}}$ is finite. Using the Born approximation $f\left(\mathbf{k}, \mathbf{k}^{\prime}\right) \approx \frac{m}{4 \pi \hbar^{2}} \tilde{U}\left(\mathbf{k}, \mathbf{k}^{\prime}\right)$, one gets:

$$
\gamma=-\frac{2 \pi \hbar^{2} a}{m} \frac{1}{(2 \pi \hbar)^{3}} \int_{\overline{\mathcal{D}}} \mathrm{d} \mathbf{k} .
$$

We stress that this approximation, well motivated here, is instead a source of divergencies if used in (38) and (41). Different approximations in (38) and (39) for the scattering amplitudes are possible since the two equations are decoupled: the latter one can be solved once the former and (40) have been solved at the same time (see the end of this subsection). Finally we observe that (40) is finite and independent on $\tilde{U}\left(\mathbf{k}, \mathbf{k}^{\prime}\right)$, and thus it does not require any further handling prior to being solved.

We consider now the explicit solution of (47), (48) and (40): we already showed that $\Delta$ and $\gamma$ cannot be non-vanishing at the same time, for this reason we consider separately the super-conductive $(\gamma=0, \Delta \neq 0)$ and normal state regimes $(\gamma \neq 0, \Delta=0)$. Notice that in the case of coexistence of superfluid and normal state, as for suitable imbalanced fermionic mixtures (see [6] and references therein), the two cases can be discussed separately.

In the superconductive regime, $\mathcal{D}$ involves all the possible momenta and $\overline{\mathcal{D}}=0$, and one has:

$$
\begin{aligned}
& \frac{m}{4 \pi \hbar^{2} a}=-\frac{1}{2} \frac{1}{(2 \pi \hbar)^{3}} \int \mathrm{d} \mathbf{k}\left(\frac{1}{\sqrt{\xi_{\mathbf{k}}^{2}+\Delta^{2}}}-\frac{1}{\varepsilon_{\mathbf{k}}}\right) \\
& n=1-\frac{1}{(2 \pi \hbar)^{3}} \int_{\mathcal{D}} \mathrm{d} \mathbf{k} \frac{\xi_{\mathbf{k}}}{E_{\mathbf{k}}},
\end{aligned}
$$

i.e., the usual equations describing the BEC/BCS crossover (see $[43,44]$ and references therein, as well as, for example, [45] for the two-dimensional case).

As occurs for (41) and (43), the parameter $\gamma$ does not appear in (49) and (50). For this reason, in the normal state, (48) can be solved independently, putting inside it the value $\tilde{\mu}$ obtained for each pair of external parameters $(a, \Omega)$, from the solution of (50) with $\Delta=0$. Here $\overline{\mathcal{D}}=\left\{\mathbf{k}:\left|\xi_{\mathbf{k}}\right|<\Omega+\gamma\right\}=$ $\left\{\mathbf{k}:\left|\frac{|\mathbf{k}|^{2}}{2 m}-\tilde{\mu}\right|<\Omega+\gamma\right\}$. The set $\overline{\mathcal{D}}$ can be rewritten as $\overline{\mathcal{D}}=\left\{\left\{\tilde{\mu}<\frac{\mathbf{k}^{2}}{2 m}<\tilde{\mu}+\Omega+\gamma\right\} \vee\{\tilde{\mu}-\right.$ $\left.\left.(\Omega+\gamma)<\frac{\mathbf{k}^{2}}{2 m}<\tilde{\mu}\right\}\right\}$, so that a straightforward integration of (48) leads to:

$$
\gamma=-\frac{4 \sqrt{2} \pi}{3 h^{3}} U V m^{\frac{3}{2}}\left[(\tilde{\mu}+\Omega+\gamma)^{\frac{3}{2}}-(\tilde{\mu}-\Omega-\gamma)^{\frac{3}{2}}\right]
$$

This equation can be solved numerically or analytically after some algebra, however the explicit solution is not very enlightening. A considerable simplification, together with a deeper insight, can be achieved neglecting the Hartree terms from the interaction $\propto a$. In this way, exploiting (35), we also obtain:

$$
\delta N=-\frac{8 \sqrt{2} \pi}{3 h^{3}} V m^{\frac{3}{2}}\left[(\mu+\Omega)^{\frac{3}{2}}-(\mu-\Omega)^{\frac{3}{2}}\right] .
$$


By direct inspection, it easy to check that (52) is equal to the imbalance between two free Fermi gases with chemical potential $\mu_{ \pm} \equiv \mu \pm \Omega$. This result, valid also in the presence of a lattice, clarifies even further the meaning of $\delta N$ and $\gamma$.

\section{Further Applications}

Various applications and extensions of the Hamiltonian (1) are interesting and experimentally feasible. In the past sections, we described two examples, based on imbalances in the initial densities of two hyperfine species in the considered mixture or in their hopping terms. In this section, we describe two possible further applications of the Rabi coupling, also feasible in present experiments.

\subsection{Time Modulation}

An interesting set of possible applications of the Rabi coupling applied to atomic mixtures opens when a time dependence on the time $\tau$ is considered in Equation (1). The resulting Hamiltonian can be mapped by the transformation in (2) to:

$$
H_{\mathrm{ROT}}(\tau)=-t \sum_{<i, j>, \alpha} a_{i \alpha}^{\dagger} a_{j \alpha}-\sum_{i}\left[(\mu-\Omega(\tau)) n_{i+}+(\mu+\Omega(\tau)) n_{i-}\right]-U \sum_{i} n_{i+} n_{i-} .
$$

This Hamiltonian can be realized in the present experiments by varying in time the intensity of the lasers inducing the Raman transitions at the basis of the Rabi coupling. The allowed profile of variation is controllable in experiments, as well as the rate of variation. It can be changed from scales much larger to those much smaller than any intrinsic timescale of the experiment [46]. In this way, different situations, including quenches or adiabatic evolutions, can be probed.

Note at the end that such a type of imbalance cannot be achieved easily without Rabi coupling acting on the numbers of atoms for each hyperfine species of the mixture, since these numbers are not easily controllable in time.

\subsection{Spatial Modulations}

Another interesting extension is the study of the space-dependence of the Rabi coupling $\Omega(i)$ in Equation (1). Again, in our knowledge such a type of imbalance cannot be achieved following other techniques. Experimentally feasible spatial dependences $\Omega(i)$ are (1) Gaussian: $\Omega\left(\mathbf{r}, \mathbf{r}_{0}\right)=\Omega e^{-\frac{\left|\mathbf{r}-\mathbf{r}_{0}\right|^{2}}{\sigma^{2}}}$, with $\sigma \approx 10-100 \mu \mathrm{m}$ and then of the order of 10-100 lattice sites for a typical lattice; and (2) sinusoidal along a direction: $\Omega(\mathbf{r})=\Omega \sin \mathbf{k}_{R} \cdot \mathbf{r}$, where $\mathbf{k}_{R}$ can be tuned varying the angle between the lasers inducing the Rabi coupling, with its maximum magnitude being $\left|\mathbf{k}_{R}\right|^{(\max )}=\frac{2 \pi}{\lambda_{R}}\left(\lambda_{R}\right.$ is the wavelength of the lasers). In this interesting case, we obtain, via the transformation in (2), a spatially-modulated chemical potential:

$$
H_{\mathrm{ROT}}(\tau)=-t \sum_{<i, j>, \alpha} a_{i \alpha}^{\dagger} a_{j \alpha}-\sum_{i}\left[\left(\mu-\Omega \cos \vec{\phi} \cdot \mathbf{r}_{i}\right) n_{i+}+\left(\mu+\Omega \cos \phi \cdot \mathbf{r}_{i}\right) n_{i-}\right]-U \sum_{i} n_{i+} n_{i-} .
$$

It would particularly interesting to study the effect of a generic modulation $\vec{\phi}$ on a superfluid phase.

As addressed at the end of Section 2, since the Rabi coupling with spatial dependence also transfers momentum $\delta \mathbf{p}$ to the atoms, in the presence of an optical lattice, its intensity is limited by the requirement that excited Wannier functions are not populated significantly [46]. The Rabi transition width involving different Wannier states reads $\Omega_{i, j} \propto\left\langle w_{i}(\mathbf{r})\left|e^{i(\delta \mathbf{p}) \cdot \mathbf{r}}\right| w_{j}(\mathbf{r})\right\rangle$, being $w_{m}(\mathbf{r})$ the $m$-th Wannier function at each site [1]. Considering just the first two Wannier functions, $w_{0}(\mathbf{r})$ and $w_{1}(\mathbf{r})$, the probability $P_{0,1}$ of transition between them by a two-photon Raman transition giving rise to the Rabi coupling is $P_{0,1}=\left(\frac{\Omega}{\sqrt{\Omega^{2}+E_{g}^{2}}}\right)^{2}, E_{g}$ being the energy difference between the two Wannier states. Imposing this quantity as much less than 1 , we obtain $\Omega \ll E_{g}$. For a deep optical lattice potential, where the confinement on the lattice is approximately harmonic, $E_{g} \sim 2 E_{R}\left(\frac{V_{0}}{E_{R}}\right)^{\frac{1}{2}}$ [1], so that 
$\frac{\Omega}{E_{R}} \lesssim 2\left(\frac{V_{0}}{E_{R}}\right)^{\frac{1}{2}}$. $V_{0}$ is the maximum intensity of the confining lattice potential and $E_{R}=\frac{\hbar^{2} k^{2}}{2 m}$ the recoil energy, $k=\frac{2 \pi}{\lambda}$ being in turn the wave vector of the laser light creating the lattice and $m$ the mass of the atoms. The lower bound for the ratio $\frac{V_{0}}{E_{R}}$, such that the tight-binding approximation holds, is $\frac{V_{0}}{E_{R}} \sim 5$. The scattering amplitude $t$ for a $n$-dimensional hyper cubic lattice in the regime $\left(\frac{V_{0}}{E_{R}}\right) \gg 1$ can be estimated as [1]

$$
t \approx \frac{4}{\sqrt{\pi}} E_{R}\left(\frac{V_{0}}{E_{R}}\right)^{\frac{3}{4}} \exp \left[-2\left(\frac{V_{0}}{E_{R}}\right)^{\frac{3}{4}}\right]
$$

From this formula, with $\frac{V_{0}}{E_{R}} \gtrsim 5$, we also obtain $\frac{t}{E_{R}} \lesssim 0.08$ and $\frac{\Omega}{t} \lesssim 23.2\left(\frac{V_{0}}{E_{R}}\right)^{\frac{1}{2}}$. We find that basically the ratio $\frac{\Omega}{t}$ is only limited by the achievable intensity for $V_{0}$ before reaching appreciable heating regimes. As written at the end of Section 2, the reachable intensities by present laser techniques can be of the same order of the Fermi energies in typical experiments, both in continuous space and on a lattice. Thus, we conclude that our method is effective in a very wide range of intensities for Rabi coupling.

\section{Conclusions}

We have shown that Rabi coupling applied to an atomic mixture of different hyperfine levels gives a simple method to design and control effective population imbalances. These effective imbalances hold for dressed one-particle states obtained diagonalizing the mixing matrix related to the Rabi coupling. The method works equally for bosonic and fermionic atoms.

The presented way of controlling the chemical potential opens up the possibility of very effectively probing the physics of interacting imbalanced mixtures. Indeed, as shown explicitly for a balanced and interacting two-species fermionic mixture, the superfluid properties in the presence of Rabi coupling are the same as those for an imbalanced mixture in the absence of the Rabi term. It would be very interesting to have spin-dependent tunneling, in order to create spin-orbit-like couplings, a case we plan to study in future work.

Notably, the proposed method can be also exploited to engineer spatially and/or temporallydependent effective population imbalances, generally not achievable in the present ultracold atoms experiments. We hope that the discussion presented in this paper will stimulate the design of new experiments in the near future.

Acknowledgments: The authors are pleased to thank Michele Burrello, Leonardo Fallani, Francesca Maria Marchetti, Massimo Mannarelli, Simone Paganelli, Guido Pagano, and Luca Salasnich for useful discussions and correspondence. A.C. acknowledges the support of the European Research Council (ERC) Synergy Grant UQUAM. L.D. acknowledges financial support from the BIRD2016 project "Superfluid properties of Fermi gases in optical potentials" of the University of Padova.

Author Contributions: Initial results on the subjects discussed in this paper were obtained in the Graduation Thesis of A.M. under the supervision of A.T.. L.L. and A.M. performed the computations presented in Sections 2 and 4. L.L. wrote Sections 3 and 5. A.C., L.D., and A.T. worked on the interpretation and on the presentation of the obtained results.

Conflicts of Interest: The authors declare no conflict of interest.

\section{References}

1. Bloch, I.; Dalibard, J.; Zwerger, W. Many-body physics with ultracold gases. Rev. Mod. Phys. 2008, 80, 885. [CrossRef]

2. Lewenstein, M.; Sanpera, A.; Ahufinger, V. Ultracold Atoms in Optical Lattices: Simulating Quantum Many-Body Systems; Oxford University Press: Oxford, UK, 2012.

3. Dalfovo, F.; Giorgini, S.; Pitaevskii, L.P.; Stringari, S. Theory of Bose-Einstein condensation in trapped gases. Rev. Mod. Phys. 1999, 71, 463. [CrossRef]

4. Pethick, C.; Smith, H. Bose-Einstein Condensation in Dilute Gases; Cambridge University Press: Cambridge, UK, 2001. 
5. Parish, M.M.; Marchetti, F.M.; Lamacraft, A.; Simons, B.D. Finite-temperature phase diagram of a polarized Fermi condensate. Nat. Phys. 2007, 3, 124-128. [CrossRef]

6. Sheehy, D.E.; Radzihovsky, L. BEC-BCS crossover, phase transitions and phase separation in polarized resonantly-paired superfluids. Ann. Phys. 2007, 322, 1790. [CrossRef]

7. Chevy, F.; Mora, C. Ultra-cold Polarized Fermi Gases. Rep. Prog. Phys. 2010, 73, 112401. [CrossRef]

8. Shin, Y.; Zwierlein, M.W.; Schunck, C.H.; Schirotzek, A.; Ketterle, W. Observation of Phase Separation in a Strongly Interacting Imbalanced Fermi Gas. Phys. Rev. Lett. 2006, 97, 030401. [CrossRef]

9. Zwierlein, M.W.; Schirotzek, A.; Schunck, C.H.; Ketterle, W. Fermionic Superfluidity with Imbalanced Spin Populations and the Quantum Phase Transition to the Normal State. Science 2006, 311, 492-496. [CrossRef]

10. Partridge, G.B.; Li, W.; Kamar, R.I.; LiBao, Y.; Hulet, R.G. Pairing and Phase Separation in a Polarized Fermi Gas. Science 2006, 311, 503-505. [CrossRef]

11. Nascimbene, S.; Navon, N.; Jiang, K.J.; Chevy, F.; Salomon, C. Exploring the thermodynamics of a universal fermi gas. Nature 2010, 463, 1057-1060. [CrossRef]

12. Mitra, D.; Brown, P.T.; Schaub, P.; Kondov, S.S.; Bakr, W.S. Phase separation and pair condensation in a spin-imbalanced 2D Fermi gas. Phys. Rev. Lett. 2016, 117, 093601. [CrossRef]

13. Yoshioka, D. The Quantum Hall Effect; Springer: New York, NY, USA, 2002.

14. Bakr, W.S.; Gillen, J.I.; Peng, A.; Fölling, S.; Greiner, M. A quantum gas microscope for detecting single atoms in a Hubbard-regime optical lattice. Nature 2009, 462, 74-77. [CrossRef]

15. Weitenberg, C.; Endres, M.; Sherson, J.F.; Cheneau, M.; Schauss, P.; Fukuhara, T.; Bloch, I.; Kuhr, S. Single-spin addressing in an atomic Mott insulator. Nature 2011, 471, 319-324. [CrossRef]

16. Williams, J.; Walser, R.; Cooper, J.; Cornell, E.; Holland, M. Nonlinear Josephson-type oscillations of a driven, two-component Bose-Einstein condensate. Phys. Rev. A 1999, 59, R31(R). [CrossRef]

17. Gupta, S.; Hadzibabic, Z.; Zwierlein, M.W.; Stan, C.A.; Dieckmann, K.; Schunck, C.H.; van Kempen, E.G.M.; Verhaar, B.J.; Ketterle, W. Radio-Frequency Spectroscopy of Ultracold Fermions. Science 2003, 300, 1723-1726. [CrossRef]

18. Smerzi, A.; Trombettoni, A.; Lopez-Arias, T.; Fort, C.; Maddaloni, P.; Minardi, F.; Inguscio, M. Macroscopic oscillations between two weakly coupled Bose-Einstein condensates. Eur. Phys. J. B 2003, 31, 457. [CrossRef]

19. Chen, Q.; He, Y.; Chien, C.-C.; Levin, K. Theory of radio frequency spectroscopy experiments in ultracold Fermi gases and their relation to photoemission in the cuprates. Rep. Prog. Phys. 2009, 72, 122501. [CrossRef]

20. Pieri, P.; Perali, A.; Strinati, G.C. Enhanced paraconductivity-like fluctuations in the radiofrequency spectra of ultracold Fermi atoms. Nat. Phys. 2009, 5, 736-740. [CrossRef]

21. Barbiero, L.; Abad, M.; Recati, A. Magnetic phase transition in coherently coupled Bose gases in optical lattices. Phys. Rev. A 2016, 93, 033645. [CrossRef]

22. Inguscio, M.; Fallani, L. Atomic Physics: Precise Measurements and Ultracold Matter; Oxford University Press: Oxford, UK, 2013.

23. Steck, D.A. Quantum and Atom Optics. Available online: http://steck.us/teaching (accessed on 11 April 2018).

24. De Salvo, B.J.; Yan, M.; Mickelson, P.G.; de Escobar, Y.M.; Killian, T.C. Degenerate Fermi gas of (87)Sr. Phys. Rev. Lett. 2010, 105, 030402. [CrossRef]

25. Tey, M.K.; Stellmer, S.; Grimm, R.; Schreck, F. Double-degenerate Bose-Fermi mixture of strontium. Phys. Rev. A 2010, 82, 011608. [CrossRef]

26. Fukuhara, T.; Takasu, Y.; Kumakura, M.; Takahashi, Y. Degenerate Fermi gases of ytterbium. Phys. Rev. Lett. 2007, 98, 030401. [CrossRef]

27. Taie, S.; Takasu, Y.; Sugawa, S.; Yamazaki, R.; Tsujimoto, T.; Murakami, R.; Takahashi, Y. Realization of a $\mathrm{SU}(2) \times \mathrm{SU}(6)$ System of Fermions in a Cold Atomic Gas. Phys. Rev. Lett. 2010, 105, 190401. [CrossRef]

28. Gorshkov, A.V.; Hermele, M.; Gurarie, V.; Xu, C.; Julienne, P.S.; Ye, J.; Zoller, P.; Demler, E.; Lukin, M.D.; Rey, A.M. Two-orbital SU(N) magnetism with ultracold alkaline-earth atoms. Nat. Phys. 2010, 6, $289-295$. [CrossRef]

29. Cazalilla, M.A.; Rey, A.M. Ultracold Fermi gases with emergent SU(N) symmetry. Rep. Progr. Phys. 2014, 77, 124401. [CrossRef]

30. Pagano, G.; Mancini, M.; Cappellini, G.; Lombardi, P.; Schäfer, F.; Hu, H.; Liu, X.-J.; Catani, J.; Sias, C.; Inguscio, M.; et al. A one-dimensional liquid of fermions with tunable spin. Nat. Phys. 2014, 10, 198-201. [CrossRef] 
31. Barros, J.C.P.; Lepori, L.; Trombettoni, A. Phase diagram and non-Abelian symmetry locking for fermionic mixtures with unequal interactions. Phys. Rev. A 2017, 96, 013603. [CrossRef]

32. Mancini, M.; Pagano, G.; Cappellini, G.; Livi, L.; Rider, M.; Catani, J.; Sias, C.; Zoller, P.; Inguscio, M.; Dalmonte, M.; et al. Observation of chiral edge states with neutral fermions in synthetic Hall ribbons. Science 2015, 349, 1510-1513. [CrossRef]

33. Lepori, L.; Trombettoni, A.; Vinci, W. Simulation of two-flavor symmetry-locking phases in ultracold fermionic mixtures. Europhys. Lett. 2015, 109, 50002. [CrossRef]

34. Giorgini, S.; Pitaevskii, L.P.; Stringari, S. Theory of ultracold atomic Fermi gases. Rev. Mod. Phys. 2008, 80, 1215. [CrossRef]

35. Stamper-Kurn, D.M.; Ueda, M. Spinor Bose gases: Symmetries, magnetism, and quantum dynamics. Rev. Mod. Phys. 2013, 85, 1191. [CrossRef]

36. Jimenez-Garcia, K.; LeBlanc, L.J.; Williams, R.A.; Beeler, M.C.; Qu, C.; Gong, M.; Zhang, C.; Spielman, I.B. Tunable Spin-Orbit Coupling via Strong Driving in Ultracold-Atom Systems. Phys. Rev. Lett. 2015, 114, 125301. [CrossRef]

37. Lepori, L.; Salasnich, L. Tunable zero and first sounds in ultracold Fermi gases with Rabi coupling. J. Stat. Mech. 2017, 2017, 043107, doi:10.1088/1742-5468/aa6580.[CrossRef]

38. Abad, M.; Recati, A. A study of coherently coupled two-component Bose-Einstein Condensates. Eur. Phys. J. D 2013, 67, 148. [CrossRef]

39. Landau, L.D.; Lifshitz, E.M. Quantum Mechanics; Elsevier: New York, NY, USA, 1981.

40. Annett, J.F. Superconductivity, Superfluids, and Condensates; Oxford University Press: Oxford, UK, 2004.

41. Pao, C.-H.; Wu, S.-T.; Yip, S.-K. Superfluid stability in BEC-BCS crossover. Phys. Rev. B 2005, 73, 132506. [CrossRef]

42. Levinsen, J.; Cooper, N.R.; Shlyapnikov, G.V. Topological px+ipy superfluid phase of fermionic polar molecules. Phys. Rev. A 2011, 84, 013603. [CrossRef]

43. Randeria, M. Bose-Einstein Condensation; Griffin, A., Snoke, D.W., Stringari, S., Eds.; Cambridge University Press: Cambridge, UK, 1995.

44. Zwerger, W. (Ed.) The BCS-BEC Crossover and Unitary Fermi Gas; Springer: New York, NY, USA, 2012.

45. Randeria, M.; Duan, J.M.; Shieh, L.Y. Superconductivity in a two-dimensional Fermi gas: Evolution from Cooper pairing to Bose condensation. Phys. Rev. B 1990, 41, 327. [CrossRef]

46. Pagano, G. University of Maryland, College Park, MD, USA. Personal communication, 2013.

(C) 2018 by the authors. Licensee MDPI, Basel, Switzerland. This article is an open access article distributed under the terms and conditions of the Creative Commons Attribution (CC BY) license (http:/ / creativecommons.org/licenses/by/4.0/). 